\section{Study of Dosimetric Properties of Flattening Filter Free Photon Beam Passing through Cadmium Free Compensator Alloy}

\author{
Kaushik S. ${ }^{1 \oplus}$, Punia R. ${ }^{2,3 * \odot}$, Tyagi A. ${ }^{4}$
}

\begin{abstract}
Background: This study aims to investigate radiation beam geometry of Cyberknife beam and change in dosimetric characteristics of six megavoltage (6MV) flattening filter free (FFF) beam after passing through high density cadmium free compensator alloy.

Material and Methods: In this experimental study, changes in FFF beam dosimetric characteristics after passing through compensator alloy was measured. Transmitted intensity of FFF beam was measured in air by an ion chamber at a source to detector distance (SDD) of $800 \mathrm{~mm}$. Extended SDD measurement also has been performed at a distance of $1270 \mathrm{~mm}$ to analyze scattering due to compensator. Linear attenuation coefficient $\left(\mu_{\text {eff }}\right)$ was measured for cadmium free compensator alloy using simple exponential attenuation model. Percentage depth doses (PDDs) have been measured by a radiation field analyzer with compensator material to observe the beam hardening and change in surface doses and depth doses.

Results: Linear attenuation coefficient of compensator alloy was measured 0.042 (Standard Deviation \pm 0.00099 ) $\mathrm{mm}^{-1}$ and it was found that there is no change with increase in collimator size. Even after increasing distance source from detector, $\mu_{\text {eff }}$ has no change. PDDs were found to increase with thickness of compensator. PDD from a $60 \mathrm{~mm}$ collimator size increased by $5 \%$ and $6 \%$ at a depth of $100 \mathrm{~mm}$ and $200 \mathrm{~mm}$, respectively in water. PDD also increased with collimator size less significantly. Surface dose was found to decrease with increase in compensator thickness.
\end{abstract}

Conclusion: Cyberknife beam has been found to be narrow beam geometry. FFF beam contains lesser scattered photons. Presence of high density compensator filters out the soft x-ray photon causes significant dosimetric changes.

Citation: Kaushik S, Punia R, Tyagi A. Study of Dosimetric Properties of Flattening Filter Free Photon Beam Passing through Cadmium Free Compensator Alloy. J Biomed Phys Eng. 2019;9(6):647-652. https://doi.org/10.31661/jbpe.v0i0.745.

\section{Keywords}

Photons; X-rays; Alloys

\section{Introduction}

I $\mathrm{n}$ modern linear accelerator (LINAC), high dose rate can be achieved in flattening filter free (FFF) X-ray photon beam [1]. The beam profiles of a FFF beam are not flat due to absence of flatting filter in the head of LINAC. Presence of flattening filter flattened the x-ray beam which otherwise peaked in forward direction and caused selective beam hardening by filtering soft component of x-ray photon beam [2]. On

${ }^{1} \mathrm{PhD}$, DRP, Guru Jambheshwar University of

Science and Technology,

Hisar, Haryana, India

${ }^{2} \mathrm{PhD}$, Maharishi Day-

anand University,

Rohtak, Haryana, India

${ }^{3} \mathrm{PhD}$, Chaudhary Ranbir

Singh University, Jind,

Haryana, India

${ }^{4} \mathrm{PhD}$, DRP, BLK Super

Speciality Hospital, New

Delhi India

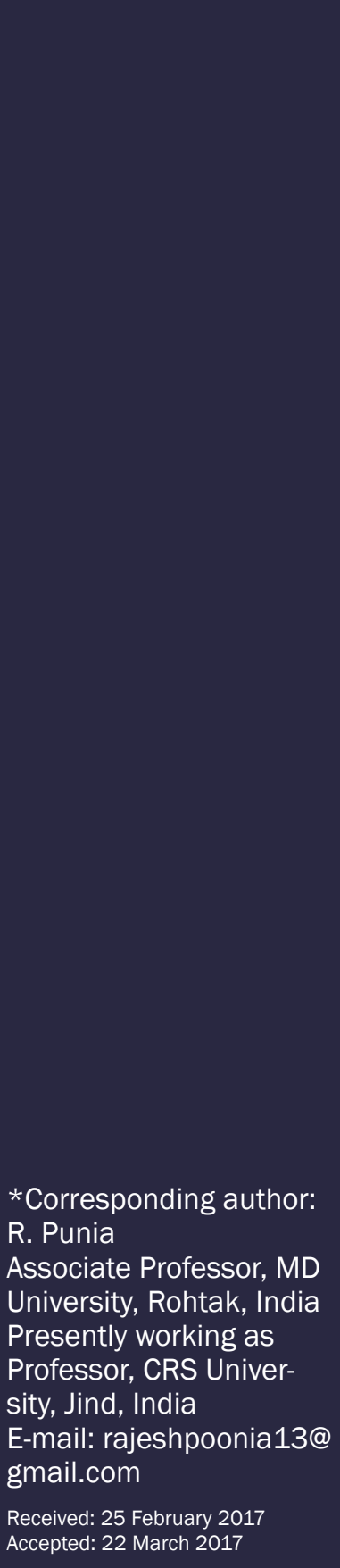

J Biomed Phys Eng 2019; 9(6) 
the other hand, $x$-ray energy spectrum of FFF beam is wider and such an unflattened beam is comprised of a reasonable amount of soft $\mathrm{x}$-ray photons [3]. Many new LINACs have come up with dual mode having option to operate with or without flattening filter. Low and high energy x-ray photon beams are usually characterized by their attenuating properties and dosimetric properties, respectively [4]. When a FFF beam is made to pass through a high density intensity modifying compensator, it changes into a reasonable hard beam. $\mathrm{Cy}$ berknife (Accuray Inc, CA, USA), a robotic radiosurgery machine produces FFF beam in which fields are defined by circular collimator with diameter from $5 \mathrm{~mm}$ to $60 \mathrm{~mm}$ [5]. Even in modern radiotherapy, compensator alloys have been used frequently [6]. Linear attenuation coefficient of a material defines thickness profile of a compensator and has been usually measured in narrow beam geometry [7]. Cyberknife beams are expected to be narrow beam geometry because of smaller collimator aperture. Therefore, present study aims to investigate the beam geometry and changes in dosimetric properties of six megavoltage (6MV) FFF photon beam of Cyberknife in the presence of high density cadmium free compensator alloy. Cadmium free compensator, a mixture of lead $(\mathrm{Pb})$, bismuth $(\mathrm{Bi})$, tin $(\mathrm{Sn})$ has been chosen for study. It has been used to deliver intensity modulated radiotherapy (IMRT) beam to treat cancer patients [8]. This mixture is dense enough to modify intensity of radiotherapy beam [9].

\section{Material and Methods}

\section{Sample Preparation}

In this experimental study, compensator slabs of cadmium free alloy were prepared by certain known percentage with weight composition of $\mathrm{Pb}, \mathrm{Sn}$ and $\mathrm{Bi}$. A total of seven thicknesses ranging from $7.1 \mathrm{~mm}$ to $61.4 \mathrm{~mm}$ were prepared. The density of said alloy is $9.802 \times$ $10^{-3} \mathrm{~g} / \mathrm{mm}^{3}$ and melting temperature is around $90-95^{\circ} \mathrm{C}$. Circular field of $6 \mathrm{MV}$ unflattened photon beam from Robotic Cyberknife VSI linear accelerator has been made to attenuate through compensator alloy. As Cyberknife does not have any tray holder like conventional linear accelerator; therefore, a slab holder was prepared and placed at a distance of $620 \mathrm{~mm}$ from x-ray source. Compensator slabs were then placed on this slab holder in air and water phantom measurements. Cyberknife fixed collimator of aperture (A) diameter, including10 $\mathrm{mm}, 20 \mathrm{~mm}, 30 \mathrm{~mm}, 40 \mathrm{~mm}, 50 \mathrm{~mm}$ and 60 $\mathrm{mm}$ were taken for attenuation and depth dose measurements. Curve fitting of the transmission and depth dose data has been performed in origin software version 8.6.

\section{Transmission}

The experimental setup for measurement of transmitted intensity of $\mathrm{x}$-ray photon is as shown in Figure 1.

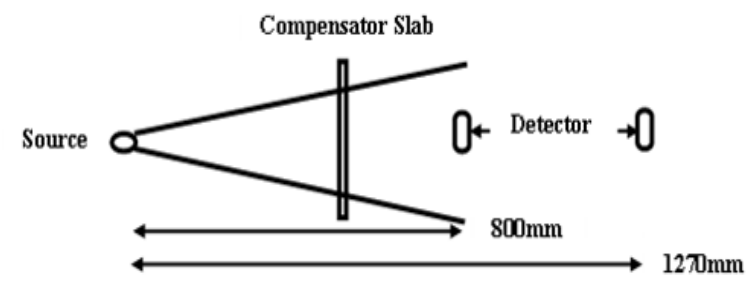

Figure 1: Experimental setup for in air transmission measurement.

FFF x-ray beam of Cyberknife was made to pass through compensator and then transmitted photon intensity has been measured by the detector at SDD of $800 \mathrm{~mm}$ and at extended SDD of $1270 \mathrm{~mm}$. In air, measurements have been made by the detector having $15 \mathrm{~mm}$ buildup cap for charge particle equilibrium. Accumulated charge for 100 monitor units (MU) was noted by Max4000 dosimeter (Standard Imaging Inc, WI, USA) and CC-13 (IBA dosimetry, Germany) ion chamber whose effective volume is $0.125 \mathrm{~cm}^{3}$. Ion chamber was placed on central axis of circular beam for all measurements. 
Percentage depth doses (PDDs)

Percentage depth dose studies have been performed by radiation field analyzer (RFA300 from IBA dosimetry). Depth dose profiles have been taken up to $280 \mathrm{~mm}$ in water by PTW TN60008 (PTW Freiburg, Germany) field and reference diode chamber. PDDs have been measured for open as well as attenuated beam through the compensator alloy of different thicknesses and collimator aperture with source to surface distance (SSD) of $800 \mathrm{~mm}$.

\section{Results}

Transmission and scatter analysis

Transmitted photon beam intensity 'I' of a mono energetic photon beam of intensity ' $\mathrm{I}_{0}$ ' through a compensator of thickness ' $t$ ' is given by simple exponential attenuation relation:

$$
\mathrm{I}=\mathrm{I}_{0} \mathrm{e}^{-\mu_{\text {eff }} \cdot \mathrm{t}}
$$

$\log I=\log I_{0}-\frac{\mu_{\text {eff }} . t}{2.303}$

Where, $\mu_{\text {eff }}$ is the linear attenuation coefficient and also depends on energy of incident photon and type of material of compensator [10]. In present study, high energy (6MV) xray photon beam has been considered as mono-energetic.

Variation of $\log$ I with thickness of compensator (t) at $800 \mathrm{~mm} \mathrm{SDD}$ and $1270 \mathrm{~mm} \mathrm{SDD}$ has been shown in Figures 2 (a) and (b). The peak photon beam intensity has been estimated from intercept of linear fitting of ' $\mathrm{log}$ I'versus't' graph. $\mu_{\text {eff }}$ has been predicted from slope of straight line graph. Experimentally measured peak intensity and peak intensity from graph have been tabulated in Tables 1 and 2 for SDD of $800 \mathrm{~mm}$ and $1270 \mathrm{~mm} \mathrm{SDD}$, respectively.

Beam hardening and surface dose

A photon beam entering a phantom is affected by a number of factors, including inverse

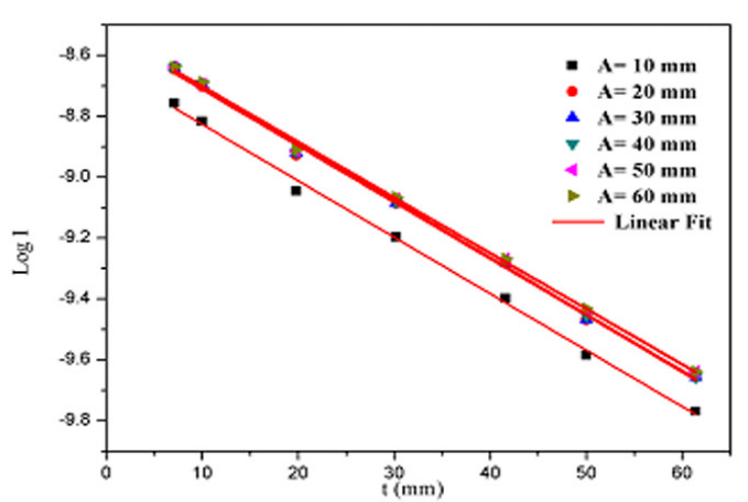

a

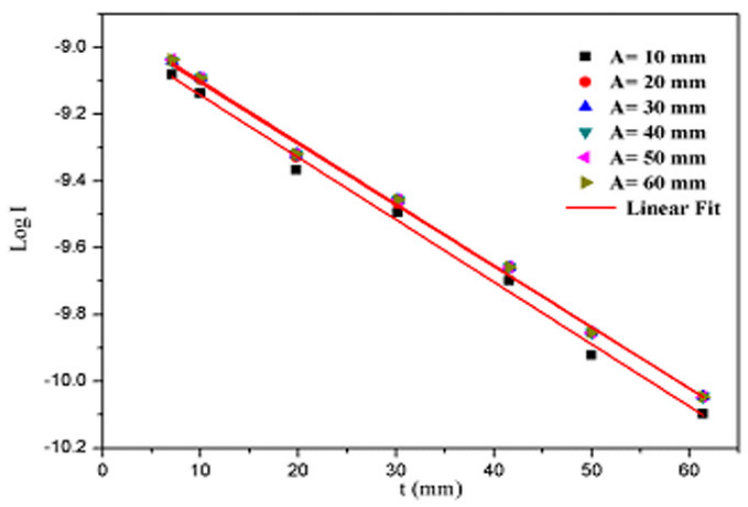

b

Figure 2: a) Variation of transmitted intensity (I) with thickness of compensator alloy at different fixed collimator aperture (A) for 800 $\mathrm{mm}$ SDD. b) Variation of transmitted intensity (I) with thickness of compensator alloy at different fixed collimator aperture (A) for $1270 \mathrm{~mm}$ SDD.

Table 1: Variation of experimentally measured maximum intensity $\left(I_{0}\right)$, maximum intensity obtained from log I vs. t graph ( $\left.\mathrm{I}_{0}{ }_{0}\right)$ and best fit parameter $\left(R_{2}\right)$ with collimator aperture (A) for $800 \mathrm{~mm}$ SDD.

\begin{tabular}{cccc}
$\mathbf{A}(\mathbf{m m})$ & $\mathbf{I}_{0}$ & $\mathbf{I}_{0}$ & $\mathbf{R}_{\mathbf{2}}$ \\
\hline $\mathbf{1 0}$ & $2.29 \mathrm{E}-09$ & $2.49 \mathrm{E}-09$ & 0.99676 \\
\hline $\mathbf{2 0}$ & $2.97 \mathrm{E}-09$ & $3.16 \mathrm{E}-09$ & 0.99739 \\
\hline $\mathbf{3 0}$ & $3.02 \mathrm{E}-09$ & $3.20 \mathrm{E}-09$ & 0.99766 \\
\hline $\mathbf{4 0}$ & $3.02 \mathrm{E}-09$ & $3.20 \mathrm{E}-09$ & 0.99789 \\
\hline $\mathbf{5 0}$ & $3.04 \mathrm{E}-09$ & $3.21 \mathrm{E}-09$ & 0.99786 \\
\hline $\mathbf{6 0}$ & $3.05 \mathrm{E}-09$ & $3.21 \mathrm{E}-09$ & 0.99813
\end{tabular}


Table 2: Variation of experimentally measured maximum intensity $\left(I_{0}\right)$, maximum intensity obtained from log I vs. $\mathrm{t}$ graph $\left(\mathrm{I}_{0}{ }_{0}\right)$ and best fit parameter $\left(R^{2}\right)$ with field sizes (D) for $1270 \mathrm{~mm}$ SDD.

\begin{tabular}{cccc}
$\mathbf{A}(\mathbf{m m})$ & $\mathbf{I}_{0}$ & $\mathbf{l}_{0}$ & $\mathbf{R}_{\mathbf{2}}$ \\
\hline $\mathbf{1 0}$ & $1.11 \mathrm{E}-09$ & $1.17 \mathrm{E}-09$ & 0.99387 \\
\hline $\mathbf{2 0}$ & $1.19 \mathrm{E}-09$ & $1.28 \mathrm{E}-09$ & 0.9957 \\
\hline $\mathbf{3 0}$ & $1.21 \mathrm{E}-09$ & $1.28 \mathrm{E}-09$ & 0.99621 \\
\hline $\mathbf{4 0}$ & $1.21 \mathrm{E}-09$ & $1.29 \mathrm{E}-09$ & 0.99621 \\
\hline $\mathbf{5 0}$ & $1.22 \mathrm{E}-09$ & $1.29 \mathrm{E}-09$ & 0.99613 \\
\hline $\mathbf{6 0}$ & $1.22 \mathrm{E}-09$ & $1.29 \mathrm{E}-09$ & 0.99613
\end{tabular}

square law, phantom attenuation and scattering [15]. Finding these components separately is a very complex phenomenon. As photon beam enters in the phantom, PDD increases from surface to depth of dose maximum $\left(\mathrm{d}_{\max }\right)$ and beyond $d_{\max }$ PDD decreases with depth in phantom. In the presence of compensator change in surface dose, depth of dose maximum and PDD beyond $\mathrm{d}_{\max }$ has been investigated.

Figure 3 shows changes in surface dose in the presence of compensator. It is evident from graph that surface dose decreases with the increase in thickness of compensator. It may be due to an increase in photon beam mean en-

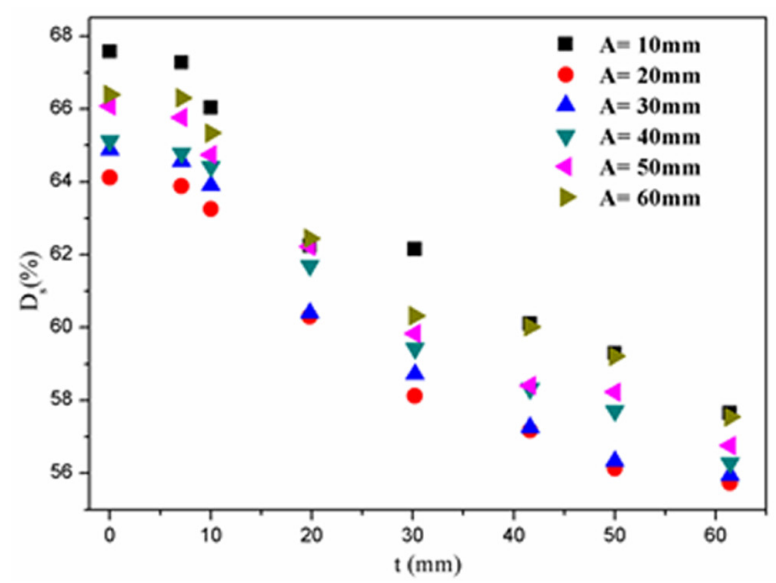

Figure 3: Variation in Surface dose $\left(D_{s}\right)$ with thickness $(t)$ of compensator alloy for different fixed collimator aperture (A). ergy derived from filtration of soft component of FFF beam. PDD data for $60 \mathrm{~mm}$ collimator opening in the presence of compensator has been presented in Figure 4 (a).

\section{Discussion}

Transmission and scatter analysis

Data presented in Tables 1 and 2 show that $\mathrm{I}_{0}$ and $\mathrm{I}_{0}$, are comparable and therefore $\mu_{\mathrm{eff}} \mathrm{ob}$ tained from linear fitting of data has been validated.

Perusal of data presented in Figures 2 (a) and (b) show that the transmitted intensity is almost same for each collimator opening expect smallest field of $10 \mathrm{~mm}$ diameter. Furthermore, slope $(\mathrm{m})$ of each line is the same i.e. 0.18;
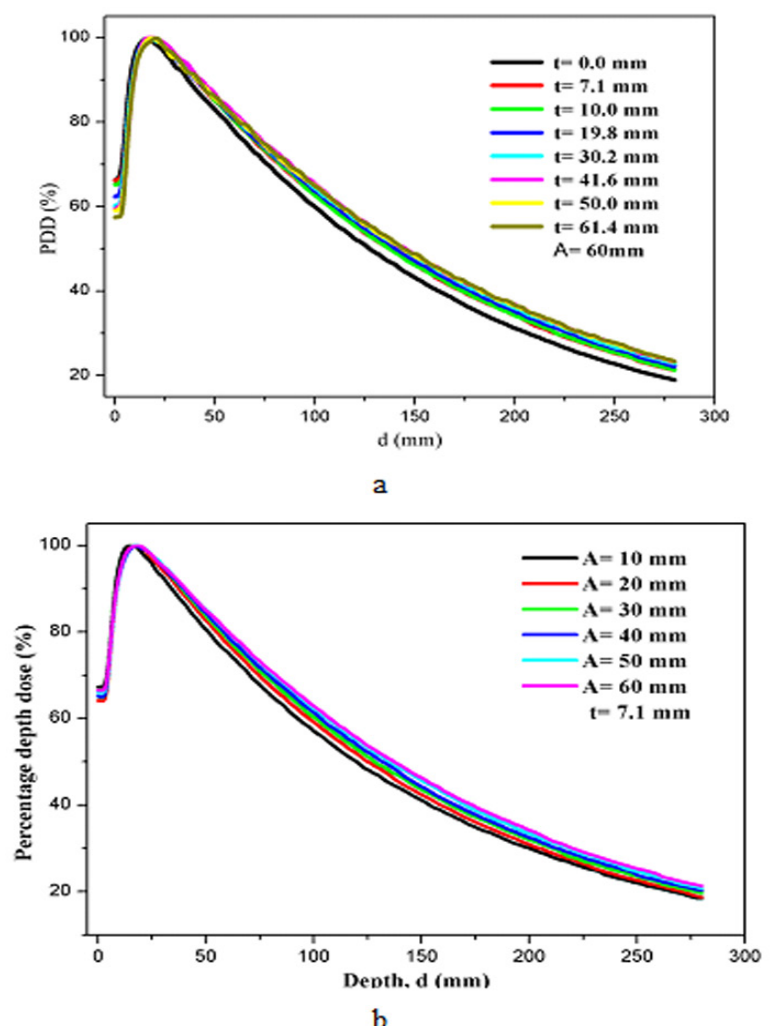

b

Figure 4: a) PDDs showing beam hardening with different compensator thickness for $A=$ $60 \mathrm{~mm}$. b) Effect of scattering due to increasing collimator opening on PDDs with $7.1 \mathrm{~mm}$ compensator. 
therefore, linear attenuation coefficient is also the same i.e. $\mu_{\text {eff }}=0.042 \mathrm{~mm}^{-1}$. From the slope, $\mu_{\text {eff }}$ has been calculated and tabulated in Table 3. Perusal of data presented in Table 3 show that an increase in collimator opening does not change the value of $\mu_{\text {eff }}$ Arora et al. [11] showed that $\mu_{\text {eff }}$ decreases with the increase in collimator opening due to an increase in scattered photon; however, our experimentally measured $\mu_{\text {eff }}$ is nearly same with

Table 3: Variation of linear attenuation coefficient $\left(\mu_{\text {eff }}\right)$ obtained from slope of log I vs. $t$ graph with collimator aperture (A) for $800 \mathrm{~mm}$ SDD and $1270 \mathrm{~mm}$ SDD.

\begin{tabular}{ccc}
$\mathbf{A}(\mathbf{m m})$ & $\boldsymbol{\mu}_{\text {eff }}\left(\mathbf{\pm 0 . 0 0 0 9 9} \mathbf{~ m m}^{-1}\right)$ measured at $\mathbf{8 0 0}$ & $\begin{array}{c}\boldsymbol{\mu}_{\text {eff }}\left(\mathbf{\pm 0 . 0 0 0 1 4} \mathbf{~ m m}^{-1}\right) \text { measured at } \mathbf{1 2 7 0} \\
\mathbf{m m} \text { SDD }\end{array}$ \\
\hline $\mathbf{1 0}$ & 0.04273 & 0.04295 \\
\hline $\mathbf{2 0}$ & 0.04272 & 0.04214 \\
\hline $\mathbf{3 0}$ & 0.04307 & 0.04236 \\
\hline $\mathbf{4 0}$ & 0.04291 & 0.04236 \\
\hline $\mathbf{5 0}$ & 0.04215 & 0.04246 \\
\hline $\mathbf{6 0}$ & 0.04212 & 0.04246
\end{tabular}

the increase in collimator opening. It may be due to narrow beam geometry of Cyberknife beam. Because a narrow beam geometry does not allow scattered photons to reach the detector. Moreover, the total scatter factor is nearly independent of collimator opening in an unflattened beam [12]. Circular beam from fixed collimator of Cyberknife unflattened beam is mainly composed of primary $\mathrm{x}$-ray photons with fewer head scattered photons as compared to a flat beam [13].

Extended source to detector distance measurements tabulated in Table 3 also shows that $\mu$ is unchanged even if compensator detector distance increases. It shows that scattered photons produced by compensator have not been reached the detector window. Results confirm that the scattered photons are very less and insignificant with the increase in collimator opening due to compensator scattering. In perfect narrow beam attenuation, the value of $\mu_{\text {eff }}$ is always independent of compensator detector distance [14]. $\mu_{\text {eff }}$ value measured in extended SDD measurement remains unchanged which indicates that the radiation beam geometry of Cyberknife is narrow beam geometry.

Beam hardening and surface dose
Perusal of data presented in Figure 4 (a) shows that $d_{\text {max }}$ is shifted towards deeper side with the increase in compensator thickness. As compensator thickness increases from 7.1 $\mathrm{mm}$ to $61.4 \mathrm{~mm}, \mathrm{~d}_{\max }$ shifts from $15 \mathrm{~mm}$ to 20 $\mathrm{mm}$. It may be towing to beam hardening in the presence of compensator alloy. Cyberknife beam is the flattening filter free beam, thus its $\mathrm{X}$-ray spectrum must contain a component of soft x-ray. For a constant SSD, field size (A), and depth in phantom (d), PDD beyond $\mathrm{d}_{\max }$ rises because of increase in beam mean energy [15]. It is understood that beyond $d_{\max }, P D D$ is rising with the increase in thickness of compensator ( $t$ ). It may be due to the increase in photon beam mean energy showing the beam hardening effect. At a depth of $100 \mathrm{~mm}$ and $200 \mathrm{~mm}$ in water, $61.4 \mathrm{~mm}$ compensator increases the PDD of $60 \mathrm{~mm}$ beam diameter by approximately $5 \%$ and $6 \%$ respectively.

For a given value of depth (d), SSD and energy of photon beam (hv), PDD is found to rise with the increase in field size because of the increase in scattered dose at point of measurement. This scattered dose accounts for collimator scattering and phantom scattering collectively. It is observed from Figure 4 (b) that PDD is increasing with collimator open- 
ing; however, it is not very significant. Because of narrow beam geometry of Cyberknife beam amount of collimator scatter is lesser which will tend to contribute lesser scattered dose to PDD. It may be the phantom scatter which mainly contributes to increase in PDD with coliimator opening. A significant amount of scattered dose may be seen in other conventional linear accelerator having larger field opening [16].

\section{Conclusion}

The compensator alloy studied is a good material to be used in radiotherapy for intensity modulation and as a compensating tissue. The measured data show that the radiation beam geometry of Cyberknife is near to narrow beam geometry. The measured dosimetric parameters characterize high-density cadmium free compensator for FFF narrow beam. Dosimetric parameters measured reveal that FFF beam contains reasonable soft component of $\mathrm{x}$-ray filtered by high density compensator causing significant beam hardening. Photon beam derived from unflattened beam contains lesser scattered photons.

\section{Acknowledgment}

We would like thank to Dr. S. Hukku and Dr. S. Halder, department of radiation oncology, Dr B L Kapur Super Speciality Hospital, New Delhi for their encouragement and moral support to work.

\section{Conflict of Interest}

\section{None}

\section{References}

1. Cashmore J. The characterization of unflattened photon beams from a 6 MV linear accelerator. Phys Med Biol. 2008;53:1933-46. doi: 10.1088/0031-9155/53/7/009. PubMed PMID: 18364548.

2. Lee PC. Monte Carlo simulations of the differential beam hardening effect of a flattening filter on a therapeutic x-ray beam. Med Phys. 1997;24:1485-9. doi: 10.1118/1.598037. PubMed PMID: 9304577.

3. Vassiliev ON, Titt U, Ponisch F, Kry SF, Mohan R, Gillin MT. Dosimetric properties of photon beams from a flattening filter free clinical accelerator. Phys Med Biol. 2006;51:1907-17. doi: 10.1088/0031-9155/51/7/019. PubMed PMID: 16552113.

4. Attix FH. Introduction to radiological physics and radiation dosimetry. 1th ed. Weinheim: Wiley-VCH; 1986. p. 222.

5. Dieterich S, Cavedon C, Chuang CF, Cohen AB, Garrett JA, Lee CL, et al. Report of AAPM TG 135: quality assurance for robotic radiosurgery. Med Phys. 2011;38:291436. doi: 10.1118/1.3579139. PubMed PMID: 21815366.

6. Dehlaghi V, Taghipour M, Haghparast A, Roshani GH, Rezaei A, Shayesteh SP, et al. Prediction of the thickness of the compensator filter in radiation therapy using computational intelligence. Med Dosim. 2015;40:53-7. doi: 10.1016/j.meddos.2014.09.003. PubMed PMID: 25498836.

7. El-Khatib EE, Podgorsak EB, Pla C. Broad beam and narrow beam attenuation in Lipowitz's metal. Med Phys. 1987;14:135-9. doi: 10.1118/1.596100. PubMed PMID: 3104738.

8. Tyagi A, Nangia S, Chufal K, Mishra M, Ghosh D, Supe $S$, et al. Quality assurance and dosimetric analysis of intensity modulation radiotherapy using compensators for head and neck cancers. Polish Journal of Medical Physics And Engineering. 2009;15:193-208. doi:10.2478/ v10013-009-0019-3.

9. Kaushik S, Punia R, Tyagi A, Malik A. Effect of scattering and differential attenuation of beam profile in the presence of high-density intensity modifying compensator. J Can Res Ther. 2019;15(8):110-4. doi: 10.4103/jcrt. JCRT_661_17.

10. Khan FM. Physics of Radiation Therapy. 3rd ed. Philadelphia: Lippincott Williams \& Wilkins; 2003. p. 60-1.

11. Arora VR, Weeks KJ. Characterization of gypsum attenuators for radiotherapy dose modification. Med Phys. 1994;21:77-81. doi: 10.1118/1.597364. PubMed PMID: 8164592.

12. Ponisch F, Titt U, Vassiliev ON, Kry SF, Mohan R. Properties of unflattened photon beams shaped by a multileaf collimator. Med Phys. 2006;33:1738-46. doi: 10.1118/1.2201149. PubMed PMID: 16872081.

13. Vassiliev ON, Titt U, Kry SF, Ponisch F, Gillin MT, Mohan R. Monte Carlo study of photon fields from a flattening filter-free clinical accelerator. Med Phys. 2006;33:820-7. doi: 10.1118/1.2174720. PubMed PMID: 16696457.

14. Huang PH, Chin LM, Bjarngard BE. Scattered photons produced by beam-modifying filters. Med Phys. 1986;13:57-63. doi: 10.1118/1.595923. PubMed PMID: 3951410.

15. Podgorsak EB. Review of radiation oncology physics: a handbook for teachers and students. International Atomic Energy Agency Educational reports series. Vienna, Austria; 2003. P. 181, 278.

16. Kaushik S, Punia R, Tyagi A, Singh MP. Dosimetric study of cadmium free alloy used in compensator based intensity modulated radiotherapy. Radiat Phys 